\title{
Interval oscillation criteria for second-order forced impulsive delay differential equations with damping term
}

\author{
Ethiraju Thandapani ${ }^{1}$, Manju Kannan ${ }^{1}$ and Sandra Pinelas ${ }^{2^{*}}$
}

${ }^{*}$ Correspondence: sandra.pinelas@gmail.com

2 Departamento De Ciencias Exactas E Naturais, Academia Militar, Av. Conde Castro Guimaraes, 2720-113 Amadora, Portugal Full list of author information is available at the end of the article

\begin{abstract}
In this paper, we present some sufficient conditions for the oscillation of all solutions of a second order forced impulsive delay differential equation with damping term. Three factors-impulse, delay and damping that affect the interval qualitative properties of solutions of equations are taken into account together. The results obtained in this paper extend and generalize some of the the known results for forced impulsive differential equations. An example is provided to illustrate the main result.
\end{abstract}

Keywords: Oscillation, Second-order, Impulse, Damping term, Differential equation Mathematics Subject Classification: 34A37, 34C10

\section{Background}

In this paper, we consider the second-order impulsive differential equation with mixed nonlinearities of the form

$$
\left\{\begin{array}{c}
\left(r(t)\left(x^{\prime}(t)\right)^{\gamma}\right)^{\prime}+p(t)\left(x^{\prime}(t)\right)^{\gamma}+q(t) x^{\gamma}(t-\delta) \\
+\sum_{i=1}^{n} q_{i}(t)|x(t-\delta)|^{\alpha_{i}-1} x(t-\delta)=e(t), \quad t \neq \tau_{k}
\end{array}\right.
$$

where $t \geq t_{0}, k \in \mathbb{N},\left\{\tau_{k}\right\}$ is the impulse moments sequence with

$$
0 \leq t_{0}=\tau_{0}<\tau_{1} \ldots, \quad \lim _{t \rightarrow \infty} \tau_{k}=\infty
$$

and

$$
\begin{aligned}
x\left(\tau_{k}\right) & =x\left(\tau_{k}^{-}\right)=\lim _{t \rightarrow \tau_{k}^{-}} x(t), x\left(\tau_{k}^{+}\right)=\lim _{t \rightarrow \tau_{k}^{+}} x(t) \\
x^{\prime}\left(\tau_{k}\right) & =x^{\prime}\left(\tau_{k}^{-}\right)=\lim _{h \rightarrow 0^{-}} \frac{x\left(\tau_{k}+h\right)-x\left(\tau_{k}\right)}{h}, \\
x^{\prime}\left(\tau_{k}^{+}\right) & =\lim _{h \rightarrow 0^{+}} \frac{x\left(\tau_{k}+h\right)-x\left(\tau_{k}^{+}\right)}{h} .
\end{aligned}
$$


Let $J \subset \mathbb{R}$ be an interval and define $P L C(J, \mathbb{R})=\{x: J \rightarrow \mathbb{R}: x(t)$ is continuous on each interval $\left(\tau_{k}, \tau_{k+1}\right), x\left(\tau_{k}^{ \pm}\right)$exist, and $x\left(\tau_{k}\right)=x\left(\tau_{k}^{-}\right)$for all $\left.k \in \mathbb{N}\right\}$.

For given $t_{0}$ and $\phi \in P L C\left(\left[t_{0}-\delta, t_{0}\right], \mathbb{R}\right)$, we say $x \in P L C\left(\left[t_{0}-\delta, \infty\right], \mathbb{R}\right)$ is a solution of Eq. (1) with initial value $\phi$ if $x(t)$ satisfies (1) for $t \geq t_{0}$ and $x(t)=\phi(t)$ for all $t \in\left[t_{0}-\delta, t_{0}\right]$. A non-trivial solution is called oscillatory if it has infinitely many zeros;otherwise it is called non-oscillatory.

In recent years the theory of impulsive differential equations emerge as an important area of research, since such equations have applications in the control theory, physics, biology, population dynamics, economics, etc. For further applications and questions concerning existence and uniqueness of solutions of impulsive differential equation, see Bainov and Simenov (1993), Lakshmikantham et al. (1989). In the last decades, interval oscillation of impulsive differential equations was arousing the interest of many researchers, see Li and Cheung (2013), Liu and Xu (2007, 2009), Muthulakshmi and Thandapani (2011) and Özbekler and Zafer $(2009,2011)$ considered the following equations

$$
\begin{cases}\left(r(t)\left(\Phi_{\alpha}\left(x^{\prime}\right)\right)^{\prime}+p(t) \Phi_{\alpha}\left(x^{\prime}\right)+q(t) \Phi_{\beta}(x)=e(t),\right. & t \neq \tau_{k} ; \\ \Delta\left(r(t) \Phi_{\alpha}\left(x^{\prime}\right)\right)+q_{i} \Phi_{\beta}(x)=e_{i}, & t=\tau_{k}, k \in \mathbb{N} .\end{cases}
$$

As far as we know, it is the first article focusing on the interval oscillation for the impulsive differential equation with damping term. Their results well improved and extended the earlier one for the equations without impulse or damping. Recently Guo et al. (2014) considered a class of second order nonlinear impulsive delay differential equations with damping term and established some interval oscillation criteria for that equation.

However, for the impulsive equations, almost all of interval oscillation results in the existing literature were established only for the case of "without delay", in other words, for the case of "with delay" the study on the interval oscillation is very scarce. To the best of our knowledge, Huang and Feng (2010) gave the first research in this direction recently. They considered second order delay differential equations with impulses

$$
\left\{\begin{array}{l}
x^{\prime \prime}(t)+p(t) f(x(t-\tau))=e(t), \quad t \geq t_{0}, \quad t \neq t_{k} ; \\
x\left(t_{k}^{+}\right)=a_{k} x\left(t_{k}\right), \quad x^{\prime}\left(t_{k}^{+}\right)=b_{k} x^{\prime}\left(t_{k}\right), \quad k=1,2, \ldots
\end{array}\right.
$$

and established some interval oscillation criteria which developed some known results for the equations without delay or impulses (Liu and Xu 2007; El Sayed 1993). It is natural to ask if it is possible to research the interval oscillation of the impulsive delay equations with damping term. In this paper, motivated mainly by Huang and Feng (2010) and Özbekler and Zafer (2009), we study the interval oscillation of second order nonlinear impulsive delay differential equations with damping term (1). We establish some interval oscillation criteria which generalize or improve some known results of Guo et al. (2012a, b, 2014), Liu and Xu (2007, 2009), Muthulakshmi and Thandapani (2011), Pandian and Purushothaman (2012), Özbekler and Zafer (2009, 2011) and Li and Cheung (2013). Finally we give an example to illustrate our main result.

\section{Main results}

Throughout this paper, we assume that the following conditions hold: 
(A1) $r(t) \in C^{1}\left(\left[t_{0}, \infty\right),(0, \infty)\right)$ and $p(t), q(t), q_{i}(t), e(t) \in P L C\left(\left[t_{0}, \infty\right), \mathbb{R}\right), i=1$, $2 \ldots, n$, with $r^{\prime}(t)+p(t) \geq 0$ for all $t \geq t_{o}$;

(A2) $\delta \geq 0, \tau_{k+1}-\tau_{k}>\delta, k \in \mathbb{N}, \alpha_{1}>\cdots>\alpha_{m}>\gamma>\alpha_{m+1}>\cdots>\alpha_{n}>0$ are constants; $a_{k}, b_{k}$ are real constants satisfying $b_{k} \geq a_{k}>0, k=1,2, \ldots$

We begin with the following notations: $I(s)=\max \left\{i: t_{0}<\tau_{i}<s\right\}, r_{j}=\max \{r(t)$ : $\left.t \in\left[c_{j}, d_{j}\right]\right\}, j=1,2$ and

$$
E_{c_{j}, d_{j}}=\left\{u \in C^{1}\left(\left[c_{j}, d_{j}\right], \mathbb{R}\right): u(t) \not \equiv 0, u\left(c_{j}\right)=u\left(d_{j}\right)=0\right\}
$$

For two constants $c, d \notin\left\{\tau_{k}\right\}$ with $c<d$ and a function $\varphi \in C([c, d], \mathbb{R})$, we define an operator $\Omega: C([c, d], \mathbb{R}) \rightarrow \mathbb{R}$ by

$$
\Omega_{c}^{d}[\varphi]= \begin{cases}0 & \text { for } \quad I(c)=I(d), \\ \varphi\left(\tau_{I(c)+1}\right) \theta(c)+\sum_{i=I(c)+2}^{I(d)} \varphi\left(\tau_{i}\right) \varepsilon\left(\tau_{i}\right) & \text { for } \quad I(c)<I(d),\end{cases}
$$

where

$$
\theta(c)=\frac{\left(a_{I(c)+1}\right)^{\gamma}-\left(b_{I(c)+1}\right)^{\gamma}}{\left(a_{I(c)+1}\right)^{\gamma}\left(\tau_{I(c)+1}-c\right)^{\gamma}} \quad \text { and } \quad \varepsilon\left(\tau_{i}\right)=\frac{a_{i}^{\gamma}-b_{i}^{\gamma}}{a_{i}^{\gamma}\left(\tau_{i}-\tau_{i-1}\right)^{\gamma}} .
$$

To prove our main results, we need the following lemmas.

Lemma 1 Let $\left(\alpha_{1}, \alpha_{2}, \ldots, \alpha_{n}\right)$ be an $n$-tuple satisfying $\alpha_{1}>\alpha_{2}>\cdots>\alpha_{m}>\gamma>$ $\alpha_{m+1}>\cdots>\alpha_{n}>0$. Then there exists an $n$-tuple $\left(\eta_{1}, \eta_{2}, \ldots, \eta_{n}\right)$ satisfying

$$
\sum_{i=1}^{n} \alpha_{i} \eta_{i}=\gamma
$$

and also either

$$
\sum_{i=1}^{n} \eta_{i}<1, \quad 0<\eta_{i}<1
$$

or

$$
\sum_{i=1}^{n} \eta_{i}=1, \quad 0<\eta_{i}<1
$$

The proof of Lemma 1 can be found in Hassan et al. (2011) and Özbekler and Zafer (2011) which is the extension of (Lemma 1, Sun and Wong 2007).

Remark 1 For given constants $\alpha_{1}>\alpha_{2}>\ldots \alpha_{m}>\gamma>\alpha_{m+1}>\cdots>\alpha_{n}>0$, Lemma 1 ensures the existence of $n$-tuple $\left(\eta_{1}, \eta_{2}, \ldots, \eta_{n}\right)$ such that either (4) and (5) or (4) and (6) hold. Particularly when $n=2$, and $\alpha_{1}>\gamma>\alpha_{2}>0$ in the first case we have 


$$
\eta_{1}=\frac{\gamma-\alpha_{2}\left(1-\eta_{0}\right)}{\alpha_{1}-\alpha_{2}}, \quad \eta_{2}=\frac{\alpha_{1}\left(1-\eta_{0}\right)-\gamma}{\alpha_{1}-\alpha_{2}}
$$

where $\eta_{0}$ be any positive number satisfying $0<\eta_{0}<\frac{\alpha_{1}-\gamma}{\alpha_{1}}$. This will ensure that $0<\eta_{1}, \eta_{2}<1$ and conditions (4) and (5) are satisfied. In the second case, we can solve (4) and (6) and obtain

$$
\eta_{1}=\frac{\gamma-\alpha_{2}}{\alpha_{1}-\alpha_{2}}, \quad \eta_{2}=\frac{\alpha_{1}-\gamma}{\alpha_{1}-\alpha_{2}} .
$$

The Lemma below can be found in Hardy et al. (1934).

Lemma 2 Let $X$ and $Y$ be non-negative real numbers. Then

$$
\lambda X Y^{\lambda-1}-X^{\lambda} \leq(\lambda-1) Y^{\lambda}, \quad \lambda>1
$$

where equality holds if and only if $X=Y$.

Let $\gamma>0, A \geq 0, B>0$ and $y>0$. Put $\lambda=1+\frac{1}{\gamma}, X=B^{\frac{\gamma}{\gamma+1}} y, Y=\left(\frac{\gamma}{\gamma+1}\right)^{\gamma} A^{\gamma} B^{\frac{-\gamma^{2}}{\gamma+1}}$ in Lemma 2, we have

$$
A-B \leq\left(\frac{A}{\gamma+1}\right)^{\gamma+1}\left(\frac{\gamma}{B}\right)^{\gamma}
$$

Theorem 1 Suppose that for any $T>0$, there exist $c_{j}, d_{j} \notin\left\{\tau_{k}\right\}, j=1,2$ such that $c_{1}<d_{1} \leq d_{1}+\delta \leq c_{2}<d_{2}$ and $q(t), q_{i}(t) \geq 0, t \in\left[c_{1}-\delta, d_{1}\right] \cup\left[c_{2}-\delta, d_{2}\right]$, $i=1,2, \ldots, n$ and

$$
e(t)= \begin{cases}\leq 0 & \text { if } t \in\left[c_{1}-\delta, d_{1}\right] \\ \geq 0 & \text { if } t \in\left[c_{2}-\delta, d_{2}\right]\end{cases}
$$

and $u_{j} \in E_{c_{j}, d_{j}}$ such that

$$
\begin{aligned}
\int_{c_{j}}^{d_{j}} & {\left[\frac{r(t)}{(\gamma+1)^{\gamma+1}}\left|(\gamma+1) u^{\prime}(t)-\frac{p(t) u(t)}{r(t)}\right|^{\gamma+1}\right] d t-\int_{c_{j}}^{\tau_{I\left(c_{j}\right)+1}} Q(t) Q_{I\left(c_{j}\right)}^{j}(t)|u(t)|^{\gamma+1} d t } \\
& -\sum_{k=I\left(c_{j}\right)+2}^{I\left(d_{j}\right)} \int_{\tau_{k-1}}^{\tau_{k}} Q(t) Q_{k}^{j}(t)|u(t)|^{\gamma+1} d t-\int_{\tau_{I\left(d_{j}\right)}}^{d_{j}} Q(t) Q_{I\left(d_{j}\right)}^{j}(t)|u(t)|^{\gamma+1} d t \\
& <r_{j} \Omega_{c_{j}}^{d_{j}}\left[|u(t)|^{\gamma+1}\right], \quad j=1,2
\end{aligned}
$$

where

$$
Q(t)=q(t)+\eta_{0}^{-\eta_{0}} \prod_{i=1}^{n}\left(\eta_{i}^{-1} q_{i}(t)\right)^{\eta_{i}}|e(t)|^{\eta_{0}}, \quad \eta_{0}=1-\sum_{i=1}^{n} \eta_{i}
$$

where $\eta_{i}>0$ are chosen according to given $\alpha_{1}, \alpha_{2}, \ldots \alpha_{n}$ as in Lemma 1 satisfying (4) and (5), and 


$$
Q_{k}^{j}(t)=\left\{\begin{array}{cl}
\frac{\left(t-\tau_{k}\right)^{\gamma}}{\left(a_{k} \delta+b_{k}\left(\tau-\tau_{k}\right)\right)^{\gamma},} & t \in\left(\tau_{k}, \tau_{k}+\delta\right), \\
\frac{\left(\tau-\tau_{k}-\delta\right)^{\gamma}}{\left(\tau-\tau_{k}\right)^{\gamma}}, & t \in\left[\tau_{k}+\delta, \tau_{k+1}\right],
\end{array} k=I\left(c_{j}\right), I\left(c_{j}\right)+1, \ldots, I\left(d_{j}\right),\right.
$$

then every solution of Eq. (1) is oscillatory.

Proof Let $x(t)$ be a non-oscillatory solution of Eq. (1). Without loss of generality, we may assume that $x(t)>0$ and $x(t-\delta)>0$ for all $t \geq t_{0}>0$. Define

$$
\omega(t)=\frac{r(t)\left(x^{\prime}(t)\right)^{\gamma}}{x^{\gamma}(t)}, \quad t \in\left[c_{1}-\delta, d_{1}\right]
$$

Then for all $t \neq \tau_{k}, t \geq t_{0}$, we have

$\omega^{\prime}(t)=-q(t) \frac{x^{\gamma}(t-\delta)}{x^{\gamma}(t)}-\sum_{i=1}^{n} q_{i}(t)|x(t-\delta)|^{\alpha_{i}-\gamma} \frac{x^{\gamma}(t-\delta)}{x^{\gamma}(t)}+\frac{e(t)}{x^{\gamma}(t)}-\frac{p(t) \omega(t)}{r(t)}-\frac{\gamma|\omega(t)|^{\frac{\gamma+1}{\gamma}}}{(r(t))^{\frac{1}{\gamma}}}$.

By taking $\eta_{0}:=1-\sum_{i=1}^{n} \eta_{i}$

$$
\begin{aligned}
\zeta_{0} & =\eta_{0}^{-1}\left|\frac{e(t) x^{\gamma}(t-\delta)}{x^{\gamma}(t)}\right| x^{-\gamma}(t-\delta) \\
\zeta_{i} & =\eta_{i}^{-1} q_{i}(t) \frac{x^{\gamma}(t-\delta)}{x^{\gamma}(t)} x^{\alpha_{i}-\gamma}(t-\delta), \quad i=1,2, \ldots, n
\end{aligned}
$$

and using the the arithmetic-geometric mean inequality,

$$
\sum_{i=0}^{n} \eta_{i} \zeta_{i} \geq \prod_{i=0}^{n} \zeta_{i}^{\eta_{i}}, \zeta_{i} \geq 0
$$

we have

$$
\begin{aligned}
\sum_{i=1}^{n} q_{i}(t) \frac{x^{\alpha_{i}-\gamma}(t-\delta)}{x^{\gamma}(t)} x^{\gamma}(t-\delta)+\frac{|e(t)|}{x^{\gamma}(t)} \geq & \eta_{0}^{-\eta_{0}}|e(t)|^{\eta_{0}} \prod_{i=0}^{n} \eta_{i}^{-\eta_{i}} q_{i}^{\eta_{i}}(t) \frac{x^{\eta_{i}\left(\alpha_{i}-\gamma\right)}(t-\delta)}{x^{\eta_{i} \gamma}(t)} x^{\eta_{i \gamma} \gamma}(t-\delta) \\
& \times \frac{x^{\eta_{0} \gamma}(t-\delta)}{x^{\eta_{0} \gamma}(t)} x^{-\eta_{0} \gamma}(t-\delta) .
\end{aligned}
$$

Since

$$
\prod_{i=0}^{n} \frac{x^{\eta_{i \gamma} \gamma}(t-\delta)}{x^{\eta_{i} \gamma}(t)}=\frac{x^{\left(\eta_{0}+\eta_{1}+\cdots+\eta_{n}\right) \gamma}(t-\delta)}{x^{\left(\eta_{0}+\eta_{1}+\cdots+\eta_{n}\right) \gamma}(t)}=\frac{x^{\gamma}(t-\delta)}{x^{\gamma}(t)}
$$

and

$$
\prod_{i=1}^{n} x^{\left(\alpha_{i}-\gamma\right) \eta_{i}}(t-\delta) x^{-\eta_{0} \gamma}(t-\delta)=1
$$


from (12), (11) becomes

$$
\begin{aligned}
\omega^{\prime}(t) & \leq-q(t) \frac{x^{\gamma}(t-\delta)}{x^{\gamma}(t)}-\eta_{0}^{-\eta_{0}} \prod_{i=1}^{n} \eta_{i}^{-\eta_{i}} q_{i}^{\eta_{i}}(t)|e(t)|^{\eta_{0}}-\frac{p(t) \omega(t)}{r(t)}-\frac{\gamma|\omega(t)|^{\frac{\gamma+1}{\gamma}}}{(r(t))^{\frac{1}{\gamma}}} \\
& =-Q(t) \frac{x^{\gamma}(t-\delta)}{x^{\gamma}(t)}-\frac{p(t) \omega(t)}{r(t)}-\frac{\gamma|\omega(t)|^{\frac{\gamma+1}{\gamma}}}{(r(t))^{\frac{1}{\gamma}}}, \quad t \neq \tau_{k} .
\end{aligned}
$$

For $t=\tau_{k}, k=1,2, \ldots$, we have

$$
\omega\left(\tau_{k}^{+}\right)=\frac{b_{k}^{\gamma}}{a_{k}^{\gamma}} \omega\left(\tau_{k}\right)
$$

Multiply both sides of (13) by $|u(t)|^{\gamma+1}$ where $u(t) \in E_{c_{1}, d_{1}}$ and integrating from $c_{1}$ to $d_{1}$, then using integration by parts on the left side, we have

$$
\begin{aligned}
& \sum_{k=I\left(c_{1}\right)+1}^{I\left(d_{1}\right)}\left|u\left(\tau_{k}\right)\right|^{\gamma+1}\left[\omega\left(\tau_{k}\right)-\omega\left(\tau_{k}^{+}\right)\right] \\
& \leq \int_{c_{1}}^{d_{1}}(\gamma+1) u^{\gamma}(t) u^{\prime}(t) \omega(t) d t-\int_{c_{1}}^{d_{1}} Q(t)|u(t)|^{\gamma+1} \frac{x^{\gamma}(t-\delta)}{x^{\gamma}(t)} d t \\
& \quad-\int_{c_{1}}^{d_{1}} \frac{p(t) \omega(t)}{r(t)}|u(t)|^{\gamma+1} d t-\int_{c_{1}}^{d_{1}} \frac{\gamma|\omega(t)|^{\frac{\gamma+1}{\gamma}}}{(r(t))^{\frac{1}{\gamma}}}|u(t)|^{\gamma+1} d t \\
& \leq-\int_{c_{1}}^{\tau_{I\left(c_{1}\right)+1}} Q(t)|u(t)|^{\gamma+1} \frac{x^{\gamma}(t-\delta)}{x^{\gamma}(t)} d t-\sum_{k=I\left(c_{1}\right)+1}^{I\left(d_{1}-1\right)} \int_{\tau_{k}}^{\tau_{k+1}} Q(t)|u(t)|^{\gamma+1} \frac{x^{\gamma}(t-\delta)}{x^{\gamma}(t)} d t \\
& \quad-\int_{\tau_{I\left(d_{1}\right)}}^{d_{1}} Q(t)|u(t)|^{\gamma+1} \frac{x^{\gamma}(t-\delta)}{x^{\gamma}(t)} d t+\int_{c_{1}}^{d_{1}}\left[\left(\left|(\gamma+1) u^{\prime}(t)-\frac{p(t) u(t)}{r(t)}\right|\right)|\omega(t)||u(t)|^{\gamma}\right. \\
& \left.\quad-\frac{\gamma|\omega(t)|^{\frac{\gamma+1}{\gamma}}}{(r(t))^{\frac{1}{\gamma}}}|u(t)|^{\gamma+1}\right] d t .
\end{aligned}
$$

Using (7) with

$$
A=\left(\left|(\gamma+1) u^{\prime}(t)-\frac{p(t) u(t)}{r(t)}\right|\right), \quad B=\frac{\gamma}{(r(t))^{\frac{1}{\gamma}}}, \text { and } y=|\omega(t)||u(t)|^{\gamma}
$$

we have

$$
\begin{aligned}
& \left((\gamma+1)\left|u^{\prime}(t)\right|-\frac{p(t)|u(t)|}{r(t)}\right)|\omega(t)||u(t)|^{\gamma}-\frac{\gamma|\omega(t)|^{\frac{\gamma+1}{\gamma}}}{(r(t))^{\frac{1}{\gamma}}}|u(t)|^{\gamma+1} \\
& \quad \leq \frac{r(t)}{(\gamma+1)^{\gamma+1}}\left((\gamma+1)\left|u^{\prime}(t)\right|-\frac{p(t)|u(t)|}{r(t)}\right)^{\gamma+1} \cdot
\end{aligned}
$$

Now for $t \in\left[c_{1}, d_{1}\right] \backslash \tau_{k}, k \in \mathbb{N}$ from (1) it is clear that $\left(r(t)\left(x^{\prime}(t)\right)^{\gamma}\right)^{\prime}+p(t)\left(x^{\prime}(t)\right)^{\gamma}=e(t)-q(t) x^{\gamma}(t-\delta)-\sum_{i=1}^{n} q_{i}(t)|x(t-\delta)|^{\alpha_{i}-1} x(t-\delta) \leq 0$. 
That is

$$
\left(\left(x^{\prime}(t)\right)^{\gamma}\right)^{\prime}+\left(\frac{r^{\prime}(t)+p(t)}{r(t)}\right)\left(x^{\prime}(t)\right)^{\gamma} \leq 0
$$

which implies that

$$
\left(x^{\prime}(t)\right)^{\gamma} \exp \int_{c_{1}}^{t} \frac{r^{\prime}(s)+p(s)}{r(s)} d s
$$

is non-increasing on $\left[c_{1}, d_{1}\right] \backslash \tau_{k}$.

Because there are different integration intervals in (15), we will estimate $x(t-\delta) / x(t)$ in each interval of $t$ as follows. We first consider the situation where $I\left(c_{1}\right) \leq I\left(d_{1}\right)$. In this case, all the impulsive moments in $\left[c_{1}, d_{1}\right]$ are $\tau_{I\left(c_{1}\right)+1}, \tau_{I\left(c_{2}\right)+1}, \ldots \tau_{I\left(d_{1}\right)}$.

Case 1 For $t \in\left(\tau_{k}, \tau_{k+1}\right] \subset\left[c_{1}, d_{1}\right]$ we have the following two sub cases:

(a) If $\tau_{k}+\delta \leq t \leq \tau_{k+1}$, then $(t-\delta, t) \subset\left(\tau_{k}, \tau_{k+1}\right]$. Thus there is no impulse moment in $(t-\delta, t)$. For any $s \in(t-\delta, t)$, we have $x(s)>x(s)-x\left(\tau_{k}^{+}\right)=x^{\prime}(\xi)\left(s-\tau_{k}\right), \quad \xi \in\left(\tau_{k}, s\right)$. Then

$(x(s))^{\gamma} \geq\left(x^{\prime}(\xi)\right)^{\gamma}\left(s-\tau_{k}\right)^{\gamma}$.

Since $\left(x^{\prime}(s)\right)^{\gamma} \exp \int_{c_{1}}^{s} \frac{r^{\prime}(v)+p(v)}{r(v)} d v$ is non-increasing in $\left[c_{1}, t\right]$, we have

$$
\left(x^{\prime}(\xi)\right)^{\gamma} \exp \int_{c_{1}}^{\xi} \frac{r^{\prime}(v)+p(v)}{r(v)} d v \geq\left(x^{\prime}(s)\right)^{\gamma} \exp \int_{c_{1}}^{s} \frac{r^{\prime}(v)+p(v)}{r(v)} d v .
$$

From (17) and (18) we have

$$
\begin{aligned}
(x(s))^{\gamma} & \geq \frac{\left(x^{\prime}(s)\right)^{\gamma} \exp \int_{c_{1}}^{s} \frac{r^{\prime}(v)+p(v)}{r(v)} d v}{\exp \int_{c_{1}}^{\xi} \frac{r^{\prime}(v)+p(v)}{r(v)} d v}\left(s-\tau_{k}\right)^{\gamma} \\
& \geq\left(x^{\prime}(s)\right)^{\gamma}\left(s-\tau_{k}\right)^{\gamma} .
\end{aligned}
$$

Therefore $\frac{x^{\prime}(s)}{x(s)}<\frac{1}{s-\tau_{k}}$. Integrating both sides of the above inequality from $t-\delta$ to $t$, we obtain

$\frac{x(t-\delta)}{x(t)}>\frac{t-\tau_{k}-\delta}{t-\tau_{k}}>0$.

(b) If $\tau_{k}<t<\tau_{k}+\delta$, then $\tau_{k}-\delta<t-\delta<\tau_{k}<t<\tau_{k}+\delta$. There is an impulsive moment $\tau_{k}$ in $(t-\delta, t)$. Similar to (a), we have $\frac{x^{\prime}(s)}{x(s)}<\frac{1}{s-\tau_{k}+\delta}$ for any $s \in\left(\tau_{k}-\delta, \tau_{k}\right]$. Upon integrating from $t-\delta$ to $\tau_{k}$, we obtain

$$
\frac{x(t-\delta)}{x\left(\tau_{k}\right)}>\frac{t-\tau_{k}}{\delta} \geq 0 .
$$

For any $t \in\left(\tau_{k}, \tau_{k}+\delta\right)$, we have

$x(t)-x\left(\tau_{k}^{+}\right)<x^{\prime}\left(t_{k}^{+}\right)\left(t-\tau_{k}\right), \xi \in\left(\tau_{k}, t\right)$. 
Using the impulsive conditions in Eq. (1) we get

$$
\begin{aligned}
x(t)-a_{k} x\left(\tau_{k}\right) & <b_{k} x^{\prime}\left(\tau_{k}\right)\left(t-\tau_{k}\right) \\
\frac{x(t)}{x\left(\tau_{k}\right)} & \leq \frac{b_{k} x^{\prime}\left(\tau_{k}\right)}{x\left(\tau_{k}\right)}\left(t-\tau_{k}\right)+a_{k} .
\end{aligned}
$$

Using $\frac{x^{\prime}\left(\tau_{k}\right)}{x\left(\tau_{k}\right)}<\frac{1}{\delta}$, we obtain

$$
\frac{x(t)}{x\left(\tau_{k}\right)}<a_{k}+\frac{b_{k}}{\delta}\left(t-\tau_{k}\right) .
$$

That is

$$
\frac{x\left(\tau_{k}\right)}{x(t)}>\frac{\delta}{a_{k} \delta+b_{k}\left(t-\tau_{k}\right)} .
$$

From (20) and (21), we have

$$
\frac{x(t-\delta)}{x(t)}>\frac{t-\tau_{k}}{a_{k} \delta+b_{k}\left(t-\tau_{k}\right)} \geq 0 .
$$

Case 2 For $t \in\left[c_{1}, \tau_{I\left(c_{1}\right)+1}\right)$ we have the following three sub-cases:

(a) If $c_{1}<t<\tau_{I\left(c_{1}\right)}+\delta$ and $\tau_{I\left(c_{1}\right)}>c_{1}-\delta$, then $t-\delta \in\left[c_{1}-\delta\right.$, $\left.\tau_{I\left(c_{1}\right)}\right)$ and there is an impulsive moment $\tau_{I\left(c_{1}\right)}$ in $(t-\delta, t)$. Similar to Case $1(\mathrm{~b})$, we have

$$
\frac{x(t-\delta)}{x(t)}>\frac{t-\tau_{I\left(c_{1}\right)}}{a_{I\left(c_{1}\right)} \delta+b_{I\left(c_{1}\right)}\left(t-\tau_{I\left(c_{1}\right)}\right)} \geq 0 .
$$

(b) If $\tau_{I\left(c_{1}\right)}+\tau<t<\tau_{I\left(c_{1}\right)+1}$ and $\tau_{I\left(c_{1}\right)}>c_{1}-\delta$, then there are no impulsive moments in $(t-\delta, t)$. Making a similar analysis of Case 1(a), we obtain $\frac{x(t-\delta)}{x(t)}>\frac{t-\delta-\tau_{I\left(c_{1}\right)}}{t-\tau_{I\left(c_{1}\right)}} \geq 0$

(c) If $\tau_{I\left(c_{1}\right)}>c_{1}-\delta$, then there are no impulsive moments in $(t-\delta, t)$. So

$$
\frac{x(t-\delta)}{x(t)}>\frac{t-\delta-\tau_{I\left(c_{1}\right)}}{t-\tau_{I\left(c_{1}\right)}} \geq 0 .
$$

Case 3 For $t \in\left(\tau_{I\left(d_{1}\right)}, d_{1}\right]$, there are three sub-cases:

(a) If $\tau_{I\left(d_{1}\right)}+\delta<d_{1}, t \in\left[\tau_{I\left(d_{1}\right)}, \tau_{I\left(d_{1}\right)}+\delta\right)$, then there is an impulsive moment $\tau_{I\left(d_{1}\right)}$. Similar to Case 2(a), we have

$$
\frac{x(t-\delta)}{x(t)}>\frac{t-\tau_{I\left(d_{1}\right)}}{a_{I\left(d_{1}\right)} \delta+b_{I\left(d_{1}\right)}\left(t-\tau_{I\left(d_{1}\right)}\right)} \geq 0 .
$$

(b) If $\tau_{I\left(d_{1}\right)}+\delta<t<d_{1}$ then there are no impulsive moments in $(t-\delta, t)$. Making a similar analysis of Case 2(b), we obtain

$$
\frac{x(t-\delta)}{x(t)}>\frac{t-\delta-\tau_{I\left(d_{1}\right)}}{t-\tau_{I\left(d_{1}\right)}} \geq 0 .
$$


(c) If $\tau_{I\left(d_{1}\right)}+\delta \geq d_{1}$, then there is an impulsive moment $\tau_{I\left(d_{1}\right)}$ in $(t-\delta, t)$.

Similar to Case 3(a), we obtain

$$
\frac{x(t-\delta)}{x(t)}>\frac{t-\tau_{I\left(d_{1}\right)}}{a_{I\left(d_{1}\right)} \delta+b_{I\left(d_{1}\right)}\left(t-\tau_{I\left(d_{1}\right)}\right)} \geq 0 .
$$

Combining all these cases, we have

$$
\frac{x^{\gamma}(t-\delta)}{x^{\gamma}(t)}>\left\{\begin{array}{lll}
Q_{I\left(c_{1}\right)}^{1}(t) & \text { for } & t \in\left[c_{1}, \tau_{I\left(c_{1}\right)+1}\right] \\
Q_{k}^{1}(t) & \text { for } & t \in\left(\tau_{k}, \tau_{k+1}\right], k=I\left(c_{1}\right)+1, \ldots, I\left(d_{1}\right)-1 \\
Q_{I\left(d_{1}\right)}^{1}(t) & \text { for } & t \in\left(\tau_{I\left(d_{1}\right)+1}, d_{1}\right]
\end{array}\right.
$$

Using (16) and (22) in (15) we get

$$
\begin{aligned}
& \sum_{k=I\left(c_{1}\right)+1}^{I\left(d_{1}\right)}\left|u\left(\tau_{k}\right)\right|^{\gamma+1}\left[\omega\left(\tau_{k}\right)-\omega\left(\tau_{k}^{+}\right)\right] \\
& \leq \int_{c_{1}}^{d_{1}} \frac{r(t)}{(\gamma+1)^{\gamma+1}}\left((\gamma+1)\left|u^{\prime}(t)\right|-\frac{p(t)|u(t)|}{r(t)}\right)^{\gamma+1} d t-\int_{c_{1}}^{\tau_{I\left(c_{1}\right)+1}} Q(t)|u(t)|^{\gamma+1} Q_{I\left(c_{1}\right)}^{1}(t) d t \\
& \quad-\sum_{k=I\left(c_{1}\right)+1}^{I\left(d_{1}-1\right)} \int_{\tau_{k}}^{\tau_{k+1}} Q(t)|u(t)|^{\gamma+1} Q_{k}^{1}(t) d t-\int_{\tau_{I\left(d_{1}\right)}}^{d_{1}} Q(t)|u(t)|^{\gamma+1} Q_{I\left(d_{1}\right)}^{1}(t) d t .
\end{aligned}
$$

For any $t \in\left(c_{1}, \tau_{I\left(c_{1}\right)+1}\right]$, we have $x(t)-x\left(c_{1}\right)=x^{\prime}(\xi)\left(t-c_{1}\right), \xi \in\left(c_{1}, t\right)$. Since $x\left(c_{1}\right)>0$, we have $x(t)>x^{\prime}(\xi)\left(t-c_{1}\right)$. Then

$$
(x(t))^{\gamma}>\left(x^{\prime}(\xi)\right)^{\gamma}\left(t-c_{1}\right)^{\gamma} .
$$

Using the monotonicity of $\left(x^{\prime}(t)\right)^{\gamma} \exp \left(\int_{c_{1}}^{t} \frac{r^{\prime}(s)+p(s)}{r(s)} d s\right)$, and (24) we have

$$
\begin{aligned}
(x(t))^{\gamma} & \geq \frac{\left(x^{\prime}(t)\right)^{\gamma} \exp \left(\int_{c_{1}}^{t} \frac{r^{\prime}(s)+p(s)}{r(s)} d s\right)}{\exp \left(\int_{c_{1}}^{\xi} \frac{r^{\prime}(s)+p(s)}{r(s)} d s\right)}\left(t-c_{1}\right)^{\gamma} \\
& \geq\left(x^{\prime}(t)\right)^{\gamma}\left(t-c_{1}\right)^{\gamma}
\end{aligned}
$$

for some $\xi \in\left(c_{1}, t\right)$. It follows

$$
\frac{\left(x^{\prime}(t)\right)^{\gamma}}{(x(t))^{\gamma}} \leq \frac{1}{\left(t-c_{1}\right)^{\gamma}} \text {. }
$$

Letting $t \rightarrow \tau_{I\left(c_{1}\right)+1}$, from (9), we have

$$
\omega\left(\tau_{I\left(c_{1}\right)+1}\right) \leq \frac{r_{1}}{\left(\tau_{I\left(c_{1}\right)+1}-c_{1}\right)^{\gamma}} .
$$

Making a similar analysis on $\left(\tau_{k-1}, \tau_{k}\right], k=I\left(c_{1}\right)+2, \ldots, I\left(d_{1}\right)$, we can prove that

$$
\omega\left(\tau_{k}\right) \leq \frac{r_{1}}{\left(\tau_{k}-\tau_{k-1}\right)^{\gamma}} .
$$


From (24), (25) and (A3), we obtain

$$
\begin{aligned}
& \sum_{k=I\left(c_{1}\right)+1}^{I\left(d_{1}\right)} \frac{a_{k}^{\gamma}-b_{k}^{\gamma}}{a_{k}^{\gamma}}\left|u\left(\tau_{k}\right)\right|^{\gamma+1} \omega\left(\tau_{k}\right) \\
& \geq \frac{a_{I\left(c_{1}\right)+1}^{\gamma}-b_{I\left(c_{1}\right)+1}^{\gamma}}{a_{I\left(c_{1}\right)+1}^{\gamma}\left(\tau_{I\left(c_{1}\right)+1}-c_{1}\right)^{\gamma}}\left|u\left(\tau_{I\left(c_{1}\right)+1}\right)\right|^{\gamma+1} r_{1}+\sum_{k=I\left(c_{1}\right)+2}^{I\left(d_{1}\right)} \frac{a_{k}^{\gamma}-b_{k}^{\gamma}}{a_{k}^{\gamma}\left(\tau_{k}-\tau_{k-1}\right)^{\gamma}}\left|u\left(\tau_{k}\right)\right|^{\gamma+1} r_{1} \\
& =r_{1} \Omega_{c_{1}}^{d_{1}}\left[|u(t)|^{\gamma+1}\right] \text {. }
\end{aligned}
$$

Since

$$
\sum_{k=I\left(c_{1}\right)+2}^{I\left(d_{1}\right)}\left|u\left(\tau_{k}\right)\right|^{\gamma+1}\left[\omega\left(\tau_{k}\right)-\omega\left(\tau_{k}^{+}\right)\right]=\sum_{k=I\left(c_{1}\right)+1}^{I\left(d_{1}\right)} \frac{a_{k}^{\gamma}-b_{k}^{\gamma}}{a_{k}^{\gamma}}\left|u\left(\tau_{k}\right)\right|^{\gamma+1} \omega\left(\tau_{k}\right),
$$

from (23) we have

$$
\begin{gathered}
\int_{c_{1}}^{d_{1}} \frac{r(t)}{(\gamma+1)^{\gamma+1}}\left((\gamma+1)\left|u^{\prime}(t)\right|-\frac{p(t)|u(t)|}{r(t)}\right)^{\gamma+1} d t-\int_{c_{1}}^{\tau_{I\left(c_{1}\right)+1}} Q(t)|u(t)|^{\gamma+1} Q_{I\left(c_{1}\right)}^{1}(t) d t \\
-\sum_{k=I\left(c_{1}\right)+2}^{I\left(d_{1}\right)-1} \int_{\tau_{k-1}}^{\tau_{k}} Q(t)|u(t)|^{\gamma+1} Q_{k}^{1}(t) d t-\int_{\tau_{I\left(d_{1}\right)}}^{d_{1}} Q(t)|u(t)|^{\gamma+1} Q_{I\left(d_{1}\right)}^{1}(t) d t>r_{1} \Omega_{c_{1}}^{d_{1}}\left[|u(t)|^{\gamma+1}\right]
\end{gathered}
$$

which contradicts $(9)$.

If $I\left(c_{1}\right)=I\left(d_{1}\right)$, then $\Omega_{c_{1}}^{d_{1}}\left[|u(t)|^{\gamma+1}\right]=0$ and there is no impulsive moments in $\left[c_{1}, d_{1}\right]$. Similar to the proof of (22), we obtain

$\int_{c_{1}}^{d_{1}} \frac{r(t)}{(\gamma+1)^{\gamma+1}}\left((\gamma+1)\left|u^{\prime}(t)\right|-\frac{p(t)|u(t)|}{r(t)}\right)^{\gamma+1} d t-\int_{c_{1}}^{\tau_{I\left(c_{1}\right)+1}} Q(t)|u(t)|^{\gamma+1} Q_{I\left(c_{1}\right)}^{1}(t) d t>0$.

It is again a contraction with our assumption. The proof when $x(t)$ is eventually negative is analogous by repeating a similar argument on the interval $\left[c_{2}, d_{2}\right]$.

Following Kong (1999) and Philos (1989), we introduce a class of functions: $D=\left\{(t, s): t_{0} \leq s \leq t\right\}, H_{1}, H_{2} \in C^{1}(D, \mathbb{R})$. A pair of functions $\left(H_{1}, H_{2}\right)$ is said to belong to a function class $\mathcal{H}$, if $H_{1}(t, t)=H_{2}(t, t)=0, H_{1}(t, s)>0, H_{2}(t, s)>0$ for $t>s$ and there exist $h_{1}, h_{2} \in L_{l o c}(D, \mathbb{R})$ such that

$$
\frac{\partial H_{1}(t, s)}{\partial t}=h_{1}(t, s) H_{1}(t, s), \quad \frac{\partial H_{2}(t, s)}{\partial s}=-h_{2}(t, s) H_{2}(t, s) .
$$

For $\lambda \in\left(c_{j}, d_{j}\right), j=1,2$,

$$
\begin{aligned}
\Gamma_{1, j}= & \int_{c_{j}}^{\tau_{I\left(c_{j}\right)+1}} H_{1}\left(t, c_{j}\right) Q(t) Q_{I\left(c_{j}\right)}^{1}(t) d t+\sum_{k=I\left(c_{j}\right)+1}^{I\left(d_{j}\right)-1} \int_{\tau_{k}}^{\tau_{k+1}} H_{1}\left(t, c_{j}\right) Q(t) Q_{k}^{1}(t) d t \\
& +\int_{\tau_{I\left(d_{j}\right)}^{d_{j}}} H_{1}\left(t, c_{j}\right) Q(t) Q_{I\left(d_{j}\right)}^{1}(t) d t \\
& -\frac{1}{(\gamma+1)^{\gamma+1}} \int_{c_{j}}^{\lambda_{j}} r(t) H_{1}\left(t, c_{j}\right)\left|h_{1}\left(t, c_{j}\right)-\frac{p(t)}{r(t)}\right|^{\gamma+1} d t,
\end{aligned}
$$


and

$$
\begin{aligned}
\Gamma_{2, j}= & \int_{\lambda_{j}}^{\tau_{I\left(\lambda_{j}\right)+1}} H_{2}\left(d_{j}, t\right) Q(t) Q_{I\left(\lambda_{j}\right)}^{1}(t) d t+\sum_{k=I\left(\lambda_{j}\right)+1}^{I\left(d_{j}\right)-1} \int_{\tau_{k}}^{\tau_{k+1}} H_{2}\left(d_{j}, t\right) Q(t) Q_{k}^{1}(t) d t \\
& +\int_{\tau_{I\left(d_{j}\right)}^{d_{j}}} H_{2}\left(d_{j}, t\right) Q(t) Q_{I\left(d_{j}\right)}^{1}(t) d t \\
& -\frac{1}{(\gamma+1)^{\gamma+1}} \int_{\lambda_{j}}^{d_{j}} r(t) H_{2}\left(t, c_{j}\right)\left|h_{1}\left(d_{j}, t\right)-\frac{p(t)}{r(t)}\right|^{\gamma+1} d t .
\end{aligned}
$$

Theorem 2 Suppose that for any $T>0$, there exist $c_{j}, d_{j}, j=1,2, \lambda \notin\left\{\tau_{k}\right\}$ such that $c_{1}<\lambda_{1}<d_{1} \leq c_{2}<\lambda_{2}<d_{2}$ and (8) holds. If there exists $\left(H_{1}, H_{2}\right) \in \mathcal{H}$ such that

$$
\frac{1}{H_{1}\left(\lambda_{1}, c_{1}\right)} \Gamma_{1,1}+\frac{1}{H_{2}\left(d_{1}, \lambda_{1}\right)} \Gamma_{2,1}>\Lambda\left(H_{1}, H_{2} ; c_{j}, d_{j}\right)
$$

where

$$
\Lambda\left(H_{1}, H_{2} ; c_{j}, d_{j}\right)=-\left[\frac{r_{j}}{H_{1}\left(\lambda_{j}, c_{j}\right)} \Omega_{c_{j}}^{\lambda_{j}}\left[H_{1}\left(., c_{j}\right)\right]+\frac{r_{j}}{H_{2}\left(d_{j}, \lambda_{j}\right)} \Omega_{\lambda_{j}}^{d_{j}}\left[H_{2}\left(d_{j}, .\right)\right]\right],
$$

then every solution of Eq. (1) is oscillatory.

Proof Let $x(t)$ be a non-oscillatory solution of Eq. (1). Proceeding as in proof of Theorem 1, we get (13) and (14). Noticing whether or not there are impulsive moments in $\left[c_{1}, \lambda_{1}\right]$ and $\left[\lambda_{1}, d_{1}\right]$, we should consider the following four cases, namely: $\quad I\left(c_{1}\right)<I\left(\lambda_{1}\right)<I\left(d_{1}\right) ; I\left(c_{1}\right)=I\left(\lambda_{1}\right)<I\left(d_{1}\right) ; I\left(c_{1}\right)<I\left(\lambda_{1}\right)=I\left(d_{1}\right) \quad$ and $I\left(c_{1}\right)=I\left(\lambda_{1}\right)=I\left(d_{1}\right)$. Moreover, in the discussion of the impulse moments of $x(t-\delta)$, it is necessary to consider the following two cases: $\tau_{I\left(\lambda_{j}\right)}+\delta>\lambda_{j}$ and $\tau_{I\left(\lambda_{j}\right)}+\delta \leq \lambda_{j}$. Here we only consider the case $I\left(c_{1}\right)<I\left(\lambda_{1}\right)<I\left(d_{1}\right)$; with $\tau_{I\left(\lambda_{j}\right)}+\delta>\lambda_{j}$. For the other cases, similar conclusions can be obtained.

For this case there are impulsive moments $\tau_{I\left(c_{1}\right)}+1, \tau_{I\left(c_{1}\right)}+2, \ldots, \tau_{I\left(\lambda_{1}\right)}$ in $\left[c_{1}, d_{1}\right]$ and $\tau_{I\left(\lambda_{1}\right)+1}, \tau_{I\left(\lambda_{1}\right)+2}, \ldots, \tau_{I\left(d_{1}\right)}$ in $\left[\lambda_{1}, d_{1}\right]$. Multiplying both sides of (13) by $H_{1}\left(t, c_{1}\right)$ and integrating it from $c_{1}$ to $\lambda_{1}$, we have

$$
\begin{aligned}
\int_{c_{1}}^{\lambda_{1}} H_{1}\left(t, c_{1}\right) Q(t) \frac{x^{\gamma}(t-\delta)}{x^{\gamma}(t)} d t \leq & -\int_{c_{1}}^{\lambda_{1}} H_{1}\left(t, c_{1}\right) \omega^{\prime}(t) d t \\
& -\int_{c_{1}}^{\lambda_{1}} \frac{p(t) \omega(t)}{r(t)} H_{1}\left(t, c_{1}\right) d t \\
& -\int_{c_{1}}^{\lambda_{1}} \frac{\gamma|\omega(t)|^{\frac{\gamma+1}{\gamma}}}{(r(t))^{\frac{1}{\gamma}}} H_{1}\left(t, c_{1}\right) d t
\end{aligned}
$$


Applying integration by parts on first integral of R.H.S of last inequality, we get

$$
\begin{aligned}
\int_{c_{1}}^{\lambda_{1}} & H_{1}\left(t, c_{1}\right) Q(t) \frac{x^{\gamma}(t-\delta)}{x^{\gamma}(t)} d t \\
\leq & -\sum_{k=I\left(c_{1}\right)+1}^{I\left(d_{1}\right)} H_{1}\left(\tau_{k}, c_{1}\right)\left(\frac{a_{k}^{\gamma}-b_{k}^{\gamma}}{a_{k}^{\gamma}}\right) \omega\left(\tau_{k}\right)-H_{1}\left(\lambda_{1}, c_{1}\right) \omega\left(\lambda_{1}\right) \\
& +\left(\int_{c_{1}}^{\tau_{I\left(c_{1}\right)+1}}+\sum_{k=I\left(c_{1}\right)+1}^{I\left(d_{1}\right)-1} \int_{\tau_{k}}^{\tau_{k+1}}+\int_{\tau_{I\left(d_{1}\right)}}^{\lambda_{1}}\right)\left[h_{1}\left(t, c_{1}\right) \omega(t)\right. \\
& -\frac{p(t)}{r(t)} \omega(t)-\frac{\gamma|\omega(t)|^{\frac{\gamma+1}{\gamma}}}{(r(t))^{\frac{1}{\gamma}}} H_{1}\left(t, c_{1}\right) d t \\
\leq & -\sum_{k=I\left(c_{1}\right)+1}^{I\left(d_{1}\right)} H_{1}\left(\tau_{k}, c_{1}\right)\left(\frac{a_{k}^{\gamma}-b_{k}^{\gamma}}{a_{k}^{\gamma}}\right) \omega\left(\tau_{k}\right)-H_{1}\left(\lambda_{1}, c_{1}\right) \omega\left(\lambda_{1}\right) \\
& +\left(\int_{c_{1}}^{\tau_{I\left(c_{1}\right)+1}}+\sum_{k=I\left(c_{1}\right)+1}^{I\left(d_{1}\right)-1} \int_{\tau_{k}}^{\tau_{k+1}}+\int_{\left.\tau_{I\left(d_{1}\right)}\right)}^{\lambda_{1}}\right)\left[h_{1}\left(t, c_{1}\right) \omega(t)\right. \\
& -\frac{p(t)|\omega(t)|^{\frac{\gamma+1}{\gamma}}}{r(t)} \mid H_{1}\left(t, c_{1}\right) d t .
\end{aligned}
$$
Using (7) with $A=\left|h_{1}\left(t, c_{1}\right)-\frac{p(t)}{r(t)}\right|, B=\frac{\gamma}{r(t)^{\frac{1}{\gamma}}}, y=|\omega(t)|$ in the last inequality, we
have

$$
\begin{aligned}
& \int_{c_{1}}^{\lambda_{1}} H_{1}\left(t, c_{1}\right) Q(t) \frac{x^{\gamma}(t-\delta)}{x^{\gamma}(t)} d t \\
& \leq-\sum_{k=I\left(c_{1}\right)+1}^{I\left(d_{1}\right)} H_{1}\left(\tau_{k}, c_{1}\right)\left(\frac{a_{k}^{\gamma}-b_{k}^{\gamma}}{a_{k}^{\gamma}}\right) \omega\left(\tau_{k}\right)-H_{1}\left(\lambda_{1}, c_{1}\right) \omega\left(\lambda_{1}\right) \\
& \quad+\frac{1}{(\gamma+1)^{\gamma+1}} \int_{c_{1}}^{\lambda_{1}} r(t) H_{1}\left(t, c_{1}\right)\left|h_{1}\left(t, c_{1}\right)-\frac{p(t)}{r(t)}\right|^{\gamma+1} d t .
\end{aligned}
$$

Similar to the proof of Theorem 1 , we need to divide the integration interval $\left[c_{1}, \lambda_{1}\right]$ into several subintervals for estimating the function $\frac{x(t-\delta)}{x(t)}$. Now,

$$
\begin{aligned}
& \int_{c_{1}}^{\lambda_{1}} H_{1}\left(t, c_{1}\right) Q(t) \frac{x^{\gamma}(t-\delta)}{x^{\gamma}(t)} d t \geq \int_{c_{1}}^{\tau_{I\left(c_{1}\right)+1}} H_{1}\left(t, c_{1}\right) Q(t) Q_{I\left(c_{1}\right)}^{1}(t) d t \\
& \quad+\sum_{k=I\left(c_{1}\right)+1}^{I\left(d_{1}\right)-1} \int_{\tau_{k}}^{\tau_{k+1}} H_{1}\left(t, c_{1}\right) Q(t) Q_{k}^{1}(t) d t+\int_{\tau_{I\left(d_{1}\right)}}^{d_{1}} H_{1}\left(t, c_{1}\right) Q(t) Q_{I\left(d_{1}\right)}^{1}(t) d t .
\end{aligned}
$$


From (31) and (32),we obtain

$$
\begin{aligned}
& \int_{c_{1}}^{\tau_{I\left(c_{1}\right)+1}} H_{1}\left(t, c_{1}\right) Q(t) Q_{I\left(c_{1}\right)}^{1}(t) d t+\sum_{k=I\left(c_{1}\right)+1}^{I\left(d_{1}\right)-1} \int_{\tau_{k}}^{\tau_{k+1}} H_{1}\left(t, c_{1}\right) Q(t) Q_{k}^{1}(t) d t \\
& \quad+\int_{\tau_{I\left(d_{1}\right)}^{d_{1}} H_{1}\left(t, c_{1}\right) Q(t) Q_{I\left(d_{1}\right)}^{1}(t) d t-\frac{1}{(\gamma+1)^{\gamma+1}} \int_{c_{1}}^{\lambda_{1}} r(t) H_{1}\left(t, c_{1}\right)\left|h_{1}\left(t, c_{1}\right)-\frac{p(t)}{r(t)}\right|^{\gamma+1} d t}^{\leq} \sum_{k=I\left(c_{1}\right)+1}^{I_{\left(\lambda_{1}\right)}} H_{1}\left(\tau_{k}, c_{1}\right)\left(\frac{a_{k}^{\gamma}-b_{k}^{\gamma}}{a_{k}^{\gamma}}\right) \omega\left(\tau_{k}\right)-H_{1}\left(\lambda_{1}, c_{1}\right) \omega\left(\lambda_{1}\right) .
\end{aligned}
$$

On the other hand multiplying both sides of (13) by $H_{2}\left(d_{1}, t\right)$ and then integrating from $\lambda_{1}$ to $d_{1}$ and using similar analysis to the above, we can obtain

$$
\begin{aligned}
& \int_{\lambda_{1}}^{\tau_{I\left(\lambda_{1}\right)+1}} H_{2}\left(d_{1}, t\right) Q(t) Q_{I\left(\lambda_{1}\right)}^{1}(t) d t+\sum_{k=I\left(\lambda_{1}\right)+1}^{I\left(d_{1}\right)-1} \int_{\tau_{k}}^{\tau_{k+1}} H_{2}\left(d_{1}, t\right) Q(t) Q_{k}^{1}(t) d t \\
& +\int_{\tau_{I\left(d_{1}\right)}}^{d_{1}} H_{2}\left(d_{1}, t\right) Q(t) Q_{I\left(d_{1}\right)}^{1}(t) d t-\frac{1}{(\gamma+1)^{\gamma+1}} \int_{\lambda_{1}}^{d_{1}} r(t) H_{2}\left(t, c_{1}\right)\left|h_{1}\left(d_{1}, t\right)-\frac{p(t)}{r(t)}\right|^{\gamma+1} d t \\
& \leq-\sum_{k=I\left(\lambda_{1}\right)+1}^{I\left(d_{1}\right)} H_{2}\left(d_{1}, \tau_{k}\right)\left(\frac{a_{k}^{\gamma}-b_{k}^{\gamma}}{a_{k}^{\gamma}}\right) \omega\left(\tau_{k}\right)-H_{2}\left(d_{1}, \lambda_{1}\right) \omega\left(\lambda_{1}\right) \text {. }
\end{aligned}
$$

Dividing (33) and (34) by $H_{1}\left(\lambda_{1}, c_{1}\right)$ and $H_{2}\left(d_{1}, \lambda_{1}\right)$ respectively and adding them, we get

$$
\begin{aligned}
& \frac{1}{H_{1}\left(\lambda_{1}, c_{1}\right)} \Gamma_{1,1}+\frac{1}{H_{2}\left(d_{1}, \lambda_{1}\right)} \Gamma_{2,1} \\
& \leq-\left(\frac{1}{H_{1}\left(\lambda_{1}, c_{1}\right)} \sum_{k=I\left(c_{1}\right)+1}^{I\left(d_{1}\right)} H_{1}\left(\tau_{k}, c_{1}\right)\left(\frac{a_{k}^{\gamma}-b_{k}^{\gamma}}{a_{k}^{\gamma}}\right) \omega\left(\tau_{k}\right)\right. \\
& \left.\quad+\frac{1}{H_{2}\left(d_{1}, \lambda_{1}\right)} \sum_{k=I\left(\lambda_{1}\right)+1}^{I\left(d_{1}\right)} H_{2}\left(d_{1}, \tau_{k}\right)\left(\frac{a_{k}^{\gamma}-b_{k}^{\gamma}}{a_{k}^{\gamma}}\right) \omega\left(\tau_{k}\right)\right) .
\end{aligned}
$$

Using the same method as in (27), we obtain

$$
\begin{aligned}
& -\sum_{k=I\left(c_{1}\right)+1}^{I\left(d_{1}\right)} H_{1}\left(\tau_{k}, c_{1}\right)\left(\frac{a_{k}^{\gamma}-b_{k}^{\gamma}}{a_{k}^{\gamma}}\right) \omega\left(\tau_{k}\right) \leq-r_{1} \Omega_{c_{1}}^{\lambda_{1}}\left[H_{1}\left(., c_{1}\right)\right] \\
& -\sum_{k=I\left(\lambda_{1}\right)+1}^{I\left(d_{1}\right)} H_{2}\left(d_{1}, \tau_{k}\right)\left(\frac{a_{k}^{\gamma}-b_{k}^{\gamma}}{a_{k}^{\gamma}}\right) \omega\left(\tau_{k}\right) \leq-r_{2} \Omega_{\lambda_{1}}^{d_{1}}\left[H_{2}\left(d_{1}, .\right)\right] .
\end{aligned}
$$

From (33) and (36), we obtain

$$
\begin{aligned}
\frac{1}{H_{1}\left(\lambda_{1}, c_{1}\right)} \Gamma_{1,1}+\frac{1}{H_{2}\left(d_{1}, \lambda_{1}\right)} \Gamma_{2,1} & \leq-\left(r_{1} \Omega_{c_{1}}^{\lambda_{1}}\left[H_{1}\left(., c_{1}\right)\right]+r_{2} \Omega_{\lambda_{1}}^{d_{1}}\left[H_{2}\left(d_{1}, .\right)\right]\right) \\
& \leq \Lambda\left(H_{1}, H_{2} ; c_{j}, d_{j}\right)
\end{aligned}
$$

which is a contradiction to the condition (29). When $x(t)<0$, we choose interval $\left[c_{2}, d_{2}\right]$ to study Eq. (1). The proof is similar and hence omitted. Now the proof is complete.

Remark 2 When $p(t)=0$, Eq. (1) reduces to the equation studied by Guo et. al (2012b). Therefore our Theorem 1 provides an extension of Theorem 2.3 with $\rho(t)=1$ to damped impulsive differential equation. 
Remark 3 When $\delta=0$, that is, the delay disappears and our results reduces to that of Theorem 2.1 and Theorem 1 with $\rho(t)=1$ in Pandian and Purushothaman (2012).

Remark 4 When $p(t)=0$ and $\gamma=1$ our Theorem 1 is a generalization of Theorem 2.2 in $\mathrm{Li}$ and Cheung (2013).

Remark 5 When the impulse is disappear, i.e., $a_{k}=b_{k}=1$ for all $k=1,2, \ldots$, the delay term $\delta=0$ and $p(t)=0$ Eq. (1) reduces to the situation studied in Hassan et al. (2011). Therefore our Theorem 1 extends Theorem 2.1 of Hassan et al. (2011).

Example 1 Consider the following impulsive differential equation

$$
\left\{\begin{array}{c}
\left(\left((2+\cos t) x^{\prime}(t) \frac{9}{5}\right)\right)^{\prime}+(1+\sin t)\left(x^{\prime}(t)\right)^{\frac{9}{5}}+m_{1}(\cos t)\left|x\left(t-\frac{\pi}{8}\right)\right|^{\frac{3}{2}} x\left(t-\frac{\pi}{8}\right) \\
\quad+m_{2}(\cos t)\left|x\left(t-\frac{\pi}{8}\right)\right|^{\frac{1}{2}} x\left(t-\frac{\pi}{8}\right)=\sin 2 t, t \neq 2 k \pi \pm \frac{\pi}{4} \\
x\left(\tau_{k}^{+}\right)=\frac{1}{3} x\left(\tau_{k}\right), x^{\prime}\left(\tau_{k}^{+}\right)=\frac{2}{3} x^{\prime}\left(\tau_{k}\right), \tau_{k}=2 k \pi \pm \frac{\pi}{4}, k=1,2, \ldots
\end{array}\right.
$$

Here $r(t)=2+$ cost, $p(t)=1+\sin t, q_{1}(t)=m_{1} \cos t, q_{2}(t)=m_{2} \cos t, e(t)=\sin 2 t$, $\gamma=\frac{9}{5}, \alpha_{1}=\frac{5}{2}, \alpha_{2}=\frac{3}{2}$ and $m_{1}, m_{2}$ are positive constants. Also $\delta=\frac{\pi}{8}, \tau_{k+1}-\tau_{k}=$ $\pi / 2>\pi / 8$. For any $T>0$, we can choose $k$ large enough such that $T<c_{1}=4 k \pi-\frac{\pi}{2}<d_{1}=4 k \pi \quad$ and $\quad c_{2}=4 k \pi+\frac{\pi}{8}<d_{2}=4 k \pi+\frac{\pi}{2}, k=1,2 \ldots$ Then there is an impulsive moment $\tau_{k}=4 k \pi-\frac{\pi}{4}$ in $\left[c_{1}, d_{1}\right]$ and an impulsive moment $\tau_{k+1}=4 k \pi+\frac{\pi}{4}$ in $\left[c_{2}, d_{2}\right]$. Now choose $\eta_{0}=1 / 5, \eta_{1}=3 / 5, \eta_{2}=1 / 5$, therefore

$$
Q(t)=5 \frac{2^{\frac{1}{5}}}{3^{\frac{3}{5}}}\left(m_{1}\right)^{\frac{3}{5}}\left(m_{2}\right)^{\frac{1}{5}}|\cos t||\sin t|^{\frac{1}{5}}
$$

If we take $u 1(t)=u_{2}(t)=\sin 4 t, \tau_{I\left(c_{1}\right)}=4 k \pi-\frac{7}{4} \pi, \tau_{I\left(d_{1}\right)}=4 k \pi-\frac{\pi}{4}$, then by a simple calculation, the left side of Eq. (9) is the following:

$$
\begin{aligned}
\int_{c_{1}}^{d_{1}} & \frac{r(t)}{(\gamma+1)^{\gamma+1}}\left((\gamma+1)\left|u^{\prime}(t)\right|-\frac{p(t)|u(t)|}{r(t)}\right)^{\gamma+1} d t-\int_{c_{1}}^{\tau_{I\left(c_{1}\right)+1}} Q(t)|u(t)|^{\gamma+1} Q_{I\left(c_{1}\right)}^{1}(t) d t \\
& -\sum_{k=I\left(c_{1}\right)+1}^{I\left(d_{1}-1\right)} \int_{\tau_{k}}^{\tau_{k+1}} Q(t)|u(t)|^{\gamma+1} Q_{k}^{1}(t) d t-\int_{\tau_{I\left(d_{1}\right)}}^{d_{1}} Q(t)|u(t)|^{\gamma+1} Q_{I\left(d_{1}\right)}^{1}(t) d t \\
\geq & \frac{1}{\left(\frac{14}{5}\right)^{\frac{14}{5}}} \int_{4 k \pi-\frac{\pi}{2}}^{4 k \pi}(2+\cos t)\left(\frac{56}{5}|\cos 4 t|-\frac{(1+\sin t)|\sin 4 t|}{(2+\cos t)}\right)^{\frac{14}{5}} d t \\
& -\int_{4 k \pi-\frac{\pi}{2}}^{4 k \pi-\frac{\pi}{4}} Q(t)|\sin 4 t|^{\frac{14}{5}}\left(\frac{t-\frac{\pi}{8}-4 k \pi+\frac{7 \pi}{4}}{t-4 k \pi+\frac{7 \pi}{4}}\right)^{\frac{9}{5}} d t \\
& -\int_{4 k \pi-\frac{\pi}{4}}^{4 k \pi-\frac{\pi}{8}} Q(t)|\sin 4 t|^{\frac{14}{5}}\left(\frac{t-4 k \pi+\frac{\pi}{4}}{a_{I\left(c_{1}\right)+1}\left(t+\frac{\pi}{8}-4 k \pi+\frac{\pi}{4}\right)}\right)^{\frac{9}{5}} d t \\
& -\int_{4 k \pi-\frac{\pi}{8}}^{4 k \pi} Q(t)|\sin 4 t|^{\frac{14}{5}}\left(\frac{t-\frac{\pi}{8}-4 k \pi+\frac{\pi}{4}}{t-4 k \pi+\frac{\pi}{4}}\right)^{\frac{9}{5}} d t \\
\approx & \left(m_{1}\right)^{\frac{3}{5}}\left(m_{2}\right)^{\frac{1}{5}}(1.5196)-0.6739 .
\end{aligned}
$$


Since $I\left(c_{1}\right)=k-1, I\left(d_{1}\right)=k, r_{1}=3$, we have

$$
\begin{aligned}
r_{1} \Omega_{c_{1}}^{d_{1}}\left[|u(t)|^{\gamma+1}\right] & =3\left|\sin 4\left(\tau_{k}\right)\right|^{\frac{14}{5}}\left(\frac{a_{k}^{\frac{9}{5}}-b_{k}^{\frac{9}{5}}}{a_{k}^{\frac{9}{5}}}\right) \\
& =0 .
\end{aligned}
$$

The condition (9) is satisfied in $\left[c_{1}, d_{1}\right]$ if

$$
\left(m_{1}\right)^{\frac{3}{5}}\left(m_{2}\right)^{\frac{1}{5}}(1.5196)<0.6739
$$

Similarly, we can show that for $t \in\left[c_{2}, d_{2}\right]$, the condition (9) is satisfied if

$$
\left(m_{1}\right)^{\frac{3}{5}}\left(m_{2}\right)^{\frac{1}{5}}(0.7553)<0.5233
$$

Since the condition (38) is weaker than (39) we can choose the constants $m_{1}, m_{2}$ small enough such that (39) holds. Hence by Theorem 1 every solution of Eq. (37) is oscillatory. In fact for $m_{1}=1 / 5, m_{2}=1 / 6$, every solution of Eq. (37) is oscillatory.

Remark 6 The result obtained in Guo et al. (2012a, b, 2014) and Erbe et al. (2010) cannot be applied to Example 1, since the results in Guo et al. (2012a) can be applicable only to equations having only one nonlinear term and the results in Guo et al. (2012b), Guo et al. (2014), Erbe et al. (2010) can be applied to equations without damping term. Therefore our results extent and compliment to Guo et al. (2012a, b, 2014), Hassan et al. (2011), Li and Cheung (2013), Pandian and Purushothaman (2012) and Erbe et al. (2010).

\section{Conclusion}

In this paper we have obtained interval oscillation criteria for Eq. (1) which extend and generalize some known results in Guo et al. (2012a), Li and Cheung (2013), Hassan et al. (2011) and Özbekler and Zafer (2011), Pandian and Purushothaman (2012).

\section{Authors' contributions}

All authors contributed equally to this paper. All authors read and approved the final manuscript.

\section{Author details}

${ }^{1}$ Ramanujan Institute for Advanced Study in Mathematics, University of Madras, Chennai 600005, India. ${ }^{2}$ Departamento De Ciencias Exactas E Naturais, Academia Militar, Av. Conde Castro Guimaraes, 2720-113 Amadora, Portugal.

\section{Acknowledgements}

The author E. Thandapani thanks University Grants Commission of India for awarding EMERITUS FELLOWSHIP [No. 6-6/2013-14/EMERITUS/-2013-14-GEN-2747/(SA-II)] to carry out this research. The author K. Manju gratefully acknowledges the Research Fellowship granted by the University Grants Commission (India) for Meritorious students in Sciences. Further the authors thank the referees for their constructive and useful suggestions which improved the content of the paper.

\section{Competing interests}

The authors declare that they have no competing interests.

Received: 25 August 2015 Accepted: 6 April 2016

Published online: 04 May 2016

\section{References}

Agarwal RP, Anderson DR, Zafer A (2010) Interval oscillation criteria for forced dealy dynamic equations with mixed nonlinearities. Comput. Math. Appl. 59:977-993 
Bainov DD, Simenov PS (1993) Impulsive differential equations: periodic solutions and applications. Longman, Harlow El Sayed MA (1993) An oscillation criteria for forced second order linear differential equations. Proc Am Math Soc 118:813-817

Erbe L, Hassan TS, Peterson A, Saker SH (2010) Interval oscillation criteria for forced second-order nonlinear delay dynamic equations with oscillatory potential. Dyn Contin Discrete Impuls Syst Ser A Math Anal 17(4):533-542

Gopalsamy K, Zhang BG (1989) On delay differential equations with impulses. J. Math. Anal. Appl. 139:1 10-122

Guo Z, Zhou X, Wang W (2014) Interval oscillation criteria for nonlinear impulsive delay differential equations with damping term. Appl. Math. Comput. 249:32-44

Guo Z, Zhou X, Wang W (2012a) Interval oscillation criteria of second order mixed nonlinear differential equations with delay. Abstr Appl Anal

Guo Z, Zhou X, Wang W (2012b) Interval oscillation criteria for super-half-linear impulsive differential equations with delay. J Appl Math

Hardy GH, Littlewood JE, Polya G (1934) Inequalities. Cambridge University Press, Cambridge

Hassan TS, Erbe L, Peterson A (2011) Forced oscillation of second order differential equations with mixed nonlinearities. Acta Math Sci 31(B):613-626

Huang M, Feng W (2010) Forced oscillation for a second order delay differential equations with impulses. Comput Math Appl 59:18-30

Kong Q (1999) Interval criteria for oscillation of second order linear differential equations. J Math Anal Appl 229:483-492

Lakshmikantham V, Bainov D, Simeonov PS (1989) Theory of impulsive differential differential equations. World Scientific Publishing Co, Singapore

Li Q, Cheung WS (2013) Interval oscillation criteria for second order delay differential equations under impulse effects. Electron J Qual Theor Diff Eq. 43:1-11

Liu X, Xu Z (2007) Oscillation of a forced super linear second order differential equation with impulses. Comput Math Appl 53:1740-1749

Liu X, Xu Z (2009) Oscillation criteria for forced mixed type Emden-Fowler equation with impulses. Appl Math Comput 215:283-291

Muthulakshmi V, Thandapani E (2011) Interval criteria for oscillation of second-order impulsive differential equation with mixed nonlinearities. Electron J Differ Eq. 2011(40):1-14

Özbekler A, Zafer A (2009) Interval criteria for the forced oscillation of super-half-linear differential equations under impulse effects. Math Comput Model 50:59-65

Özbekler A, Zafer A (2011) Oscillation of solutions of second order mixed nonlinear differential equations under impulsive perturbations. Comput Math Appl 61:933-940

Pandian S, Purushothaman G (2012) Interval Oscillation criteria for second order mixed nonlinear forced impulsive differential equations with damping term. Diff Equ Appl 4:371-387

Philos G (1989) Oscillation theorems for linear differential equations of second order. Arch Math 53:482-492

Sun YG, Wong JSW (2007) Oscillation criteria for second order forced ordinary differential equations with mixed nonlinearities. J Math Anal Appl 334:549-560

Xiaoliang Z, Zhonghai G, Wu-Sheng W (2014) Interval oscillation criteria for nonlinear impulsive delay differential equations with damping term. Appl Math Comput 249:32-44

Xiaoliang Z, Zhonghai G, Wu-Sheng W (2012a) Interval oscillation criteria for super-half-linear impulsive differential equations with delay. J Appl Math

Xiaoliang Z, Zhonghai G, Wu-Sheng W (2012b) Interval oscillation criteria of second order mixed nonlinear impulsive differential equations with delay. Abstr Appl Anal

\section{Submit your manuscript to a SpringerOpen ${ }^{\circ}$ journal and benefit from:}

- Convenient online submission

- Rigorous peer review

- Immediate publication on acceptance

- Open access: articles freely available online

- High visibility within the field

- Retaining the copyright to your article

Submit your next manuscript at $\boldsymbol{\nabla}$ springeropen.com 